\title{
Assessment Report on Anti-Rabies Dogs Vaccination Performance in Four Sub-Cities of the Addis Ababa City Administration
}

\author{
Tayech Bereket $^{1} \quad$ Tigist Kabtimer ${ }^{2}$ \\ 1.Addis Ababa City Government Farmers and Urban Agricultural Development Commission P.O. Box 3328, \\ Ethiopia \\ 2.Veterinary Drug and Animal Feed Administration Control Authority P.O.Box31303, Ethiopia
}

\begin{abstract}
Rabies is a deadly zoonotic disease with world-wide occurrence, and is transmitted through bite. Many of the victims are children, more likely to play with or approach Free Roaming Dogs. Despite rabies has remained neglected by relevant veterinary, medical and public authorities. Addis Ababa is the capital city of Ethiopia. The number of free roaming canidae and apparently, loose management of dogs contributes to a high endemicity of canine rabies in the capital city. The anti-rabies mass vaccination was carried from November 2007 to January 2010 a total 1070 owned dogs in 4 major Sub-cities; covering; Gulele (70 \%), Addis Ketema(12\%), Arada(10\%), and Kolfe-Keranio, (8\%) within the Addis Ababa City Administration that encompasses 116 Woredas. During the same period were vaccinated, of which male dogs were $700(65 \%)$ and females $370(35 \%)$ respectively. The age structure ranged from 1 month to 19 years, of which $367(34.3 \%)$ were less than 1 year $(\leq 1$ year), 623 dogs $(58.2 \%)$ being between one to ten years (1-10 years) and the remaining 80 dogs (7.5\%) were above 10 years ( $\geq 10$ years), with an average age structure of 4 years and 5 months. From the present vaccination data it was interesting to observe that the dominant colors among 1070 immunized canines were brown,(460) 43\%), and black (265) $24.8 \%$, while the remaining population being; white (110 ) $10.3 \%$, grey (70) $6.3 \%$, and other different colors (165) $15.4 \%$ respectively. The reporter also noted that among the vaccinated canines about $80 \%$ revealed to be indigenous, while the remaining were mongrels or hybrids of unidentified breeds. This is an indication of absence of breeding policy. The vaccination was being carried out as a regular annual activity for possible minimization of the transmission of the fatal disease for the well-being of public health.
\end{abstract}

Keywords: Canine, Dog, Rabies, Vaccination

DOI: $10.7176 / \mathrm{JBAH} / 9-21-07$

Publication date: November $30^{\text {th }} 2019$

\section{INTRODUCTION}

Rabies is a deadly zoonotic disease with world-wide occurrence, caused by a virus of the family Rhabdoviridae and the genus Lyssavirus. The antigenic structures of Rabies Virus biotypes are stable in nature and not easily affected by passage in laboratory hosts or cell cultures.

Rabies is responsible for estimated annual human mortalities of 31,000 and 24,000 in Asia and Africa, respectively, with people mostly at risk of dying due to rabies being those who live in rural areas of these continents (JVMAH, 2014). It is far below the 70 percent needed to halt the transmission of canine rabies in Ethiopia. Rabies as an important social and economic factors has not been given due considerations by the relevant veterinary, medical and public authorities. WHO records indicate that $40 \%$ of people are children between the ages 5-14 years who are bitten by suspect rabid animals. In up to $99 \%$ of cases, domestic dogs are responsible for rabies virus. Treating a rabies exposure, where the average cost of rabies post-exposure prophylaxis (PEP) is \$US 40 in Africa, and $\$ 49$ in Asia, can be a catastrophic financial burden on affected families whose average daily income is around \$US 1-2 per person.

Data recorded at the Ethiopian Health and Nutrition Research Institute (EHNRI, 2001) that occurrence of rabies in other domestic animals and wild fauna could be due to spillovers of infections from canine rabies. In Ethiopia, the national annual estimates from official reports indicate 12 exposure cases per 100,000 population and 1.6 rabies deaths per 100,000 populations. However, the actual numbers are expected to be higher as many cases are not reported. According to, the Ethiopian Health and Nutrition Research Institute (EHNRI, 2011), from the total of 2667 brain samples examined from dogs during 1999-2000, 1951 (73.2\%) were positive for rabies. Dogs accounted to $96.2 \%$ of the total animals examined and represented $89.83 \%$ of the total brain samples that were found to be laboratory conformed positive rabies cases. During the indicated period cats accounted for $5.35 \%$ of the total confirmed rabies cases and represented $2.62 \%$ of the total brain samples. The above facts illustrate that rabies is well established in Ethiopia, Addis Ababa, in particular.

Although Rabies has been recognized as one of the public health issues, people's awareness about what to do if bitten by dogs is low, and most of them often do not seek medical help when bitten. According to the USACDC-Information, 2017, each year thousands of people are infected with rabies in Ethiopia and an estimated 2,700 people die, which is one of the highest rabies death rates in the world. However, the true number of death caused by rabies is unknown because the disease is underreported and rabies diagnostic laboratories are not established. 
To the author's knowledge, the human risk for rabies is directly linked to the high population in dogs, and like many other countries with high human rabies death rate, the rabies vaccination coverage among dogs is very low in Ethiopia.

Rabies remains endemic in Addis Ababa and as a whole in Ethiopia, and it is indeed representing a serious veterinary and public health problem. Therefore, this report aimed to seize an opportunity to address the importance of the disease and express optimistic professional views that concerned stakeholders should take appropriate measure in developing a clear policy and strategy to control rabies in Addis Ababa and Ethiopia as a whole.

\section{General Objectives}

The report attempts to indicate the outcome of the vaccination program, importance of the disease and its prevalence as well

\section{Specific objective}

- Assessment and documentation of the outcome of the vaccination program and the potential risk factors of canine rabies based on evidence.

- Sharing of professional views for major actors and make recommendations for policy intervention

\section{Description of Program Coverage Sites}

The Sub-cities lie at an altitude between 2,300-2,500 meters above sea level with an average temperature ranging between $8.9^{\circ} \mathrm{C}-24.42^{\circ} \mathrm{C}$, with $77 \%$ average humidity. According to the CSA, (2012), the human population of the aforementioned 4 Sub-cities as of 2011 was the following: Gulele : 248,865, Addis Ketema : 271,644, Arada : 225,999 and Kolfe Keranio: 546,219, with the total estimation of 1.3 million.

The anti-rabies mass vaccination was carried out in 4 major Sub-cities, that covered Gulele (75\%), Addis Ketema (12\%), Arada (10.5\%), including Kolfe-Keranio (8\%), within the Addis Ababa City Administration that encompass 116 Woredas. The vaccination was carried out from November 2007 to January 2010. It is also interesting to observe that among the vaccinated dogs the dominant colors were of brown (460 or $43 \%$ ), following black ( 265 or $24.8 \%)$, white (110 or $10.3 \%)$, and grey ( 70 or $6.5 \%)$ and the remaining (165 or $15.4 \%)$ had been of different colors.

\section{Demographic Characteristics of Vaccinated Dogs}

The number of vaccinated dogs during the period of 2 years (November 2007-January 2010) ,as shown in Table 1,totals 1070, of which Male dogs accounts for $65 \%$ (700) and females 35\% (370) respectively.

The age structure ,depicted in Table 1, ranged from 1 month to 19 years, of which 367 (34.3\%) were less than 1 year ( $\leq 1$ year),623 dogs (58.2\%) being between one to ten years (1-10 years) and the remaining 80 dogs $(7.5 \%)$ were above 10 years ( $\geq 10$ years), with an average age structure of 4 years and 5 months. Among the vaccinated canines about $80 \%$ revealed to be indigenous (exotics) while the remaining were mongrels or hybrids of unidentified breeds (Table 1).

Table 1: Demographic Characteristics of Vaccinated Dogs

\begin{tabular}{|c|c|c|c|c|c|c|c|c|c|c|}
\hline $\begin{array}{l}\text { Characteristics/ } \\
\text { Sub-city }\end{array}$ & Gulele & $\%$ & $\begin{array}{l}\text { Addis } \\
\text { Ketema }\end{array}$ & $\%$ & Arada & $\%$ & $\begin{array}{l}\text { Kolfe - } \\
\text { Keranio }\end{array}$ & $\%$ & Total & $\%$ \\
\hline \multicolumn{11}{|l|}{ 1.Sex } \\
\hline 1.1 Male & 490 & 65.4 & 84 & 65.6 & 70 & 65.4 & 56 & 65 & 700 & 65 \\
\hline 1.2 Female & 259 & 34.6 & 44 & 34.4 & 37 & 34.6 & 30 & 35 & 370 & 35 \\
\hline Sub-total & 749 & 100 & 128 & 100 & 107 & 100 & 86 & 100 & 1070 & \\
\hline \multicolumn{11}{|l|}{ 2.Age Category } \\
\hline$\leq 1 \mathrm{yr}$ & 257 & 34.3 & 44 & 34.1 & 37 & 34.6 & 29 & 34.1 & 367 & 34.3 \\
\hline $1-10$ yrs. & 436 & 58.2 & 75 & 58.1 & 62 & 58 & 50 & 58.8 & 623 & 58.2 \\
\hline $1.4 \geq 10$ yrs. & 56 & 7.5 & 10 & 7.8 & 8 & 7.4 & 6 & 7.1 & 80 & 7.5 \\
\hline Sub-total & 749 & 100 & 129 & 100 & 107 & 100 & 85 & 100 & 1070 & 100 \\
\hline \multicolumn{11}{|l|}{ 3.Breed } \\
\hline 1.5 Exotic & 599 & 80 & 103 & 80 & 86 & 80.3 & 68 & 80 & 856 & 80 \\
\hline 1.6 Mongrel/Hybrid & 150 & 20 & 26 & 20 & 21 & 19.7 & 17 & 20 & 214 & 20 \\
\hline Sub-total & 749 & 100 & 129 & 100 & 107 & 100 & 85 & 100 & 1070 & 100 \\
\hline
\end{tabular}

It is also interesting to observe that among the vaccinated dogs, the dominant colors were of brown (460 or $43 \%$ ), following black (265 or $24.8 \%$ ), white (110 or $10.3 \%$ ), and grey ( 70 or $6.5 \%$ ) and the remaining (165 or $15.4 \%$ ) had been of different colors. 


\section{DISCUSSION}

Rabies is a preventable viral disease most often transmitted through the bite of a rabid animal. The rabies virus infects the central nervous system (CNS) of mammals, ultimately causes disease in the brain and death.

In Ethiopia, priority has been given to diseases like Malaria, HIV/AIDS and TB that attract funding for achieving Millennium Development Goals (MDG). In addition, these diseases are rated as top priority in the current Ethiopian Growth and Transformation Plan (GTP). According the WHO recommendation, vaccinating $70 \%$ of the dog population helps to control rabies, and thus, prevent the rabies virus from circulating amongst susceptible animals. Vaccination of domestic dogs is a highly recommended strategy to prevent and control rabies. However, culling, which is an immediate and visible response to public concerns about rabies, is still frequently carried out in response to rabies outbreaks, which is an indication of an inadequate practice of vaccinating domestic dogs as a strategy to control rabies. Eshetu et al (2003) reported that the total number of owned dogs in Addis Ababa was estimated at 225,078, which may be an underestimate of the actual population as it only considered four months of the year. The study showed that about 33\% of dog owners brought their dogs for vaccination, and $67.6 \%$ of owners keep their dogs in well fenced houses. 1070 dogs were vaccinated from November 2007 to January 2010 against rabies in 116 Woredas of Gulele, Addis Ketema, Kolfe - Keranio and Arada Sub-cities of the Addis Ababa City Administration. As depicted in Table 1, the age stratification ranged from 1 month to19 years, of which $367(34.3 \%)$ were less than 1 year ( $\leq 1$ year) ,623 dogs $(58.2 \%)$ being between one to ten years (1-10 years) and the remaining smaller population 80 dogs or $7.5 \%$ were above 10 years ( $\geq 10$ years). The reporter also observed that among the vaccinated canines, about $80 \%$ revealed to be indigenous (exotics) while about $20 \%$ were mongrels or hybrids of unidentified breeds (Table 1). Although Rabies is not comparable with these diseases which cause very high health and economic burden, one can take into account that Rabies causes a health economic hardship and with a high likelihood of fatality for exposed rural inhabitants calling for national and global attention.

Considering the goal set by WHO to eliminate dog-mediated human rabies by 2030, and meeting the Millennium Development Goals (MDG), list of combating disease burden and reducing extreme poverty, including rabies control into the Ethiopian Growth and Transformation Plan (GTP) would be very crucial.

\section{CONCLUSIONS AND RECOMMENDATIONS}

The present report revealed that dogs' owners are willing to bring their dogs for vaccination if conditions are conducive. Based on the findings of the report the human risk for rabies is directly linked to the high population in dogs following high human rabies death rate.

Mass vaccination was covered by Gulele (75 \%) followed by Addis Ketema (12\%), Arada (10.5\%) and KolfeKeranio (8\%), within four Sub-cities of Addis Ababa City Administration

When taking place to compare the number of vaccinated dogs out of 1070,of which Male dogs accounts for $65 \%$ and females $35 \%$ ( 370$)$.

The age structure ranged from 1 month to 19 years, of which $34.3 \%$ were less than 1 year $58.2 \%$ being between one to ten years and the remaining $7.5 \%$ were above 10 years, with an average age structure of 4 years and 5 months.

Among the vaccinated canines about $80 \%$ revealed to be indigenous (exotics) while the remaining were mongrels or hybrids of unidentified breed

According to the World Health Organization (WHO), vaccinating 70\% of the dog population in any given area helps to control and prevent rabies virus in human population.

Based the above conclusions the following recommendations forwards

Addis Ababa being the Capital of Ethiopia and Africa, there is an urgent need for a coordinated rabies control activities by forming a sustainable standing committee or any other relevant body. A successful pilot rabies control program in Addis Ababa could serve as a model to cover other urban and rural areas in Ethiopia;

- The role of media was found to be low in disseminating information to the public about this deadly but completely preventable disease;

- Therefore, strong extension services should be in place, and in the mean time regular vaccination campaigns, possibly 70 percent and above coverage, should be combined with continuing vaccination scheme for young dogs;

- Animal health services is a public good, government involvement in the sector should be reduced as far as possible. It is apparent that private sector is more effective than the government in providing the necessary services in animal health in general;

- Further epidemiological investigation is required to fully understand the extent of the distribution of the disease in other parts of the country.

- It is indeed a time to initiate and develop a clear policy and legislation with adequate enforcement requiring the registration, licensing and taxation of dogs, a measure which is often considered as the basis for mass immunization and dog population control. 


\section{REFERENCES}

$10^{\text {th }}$ World Rabies Day: Educate-Vaccinate -Eliminate, September 2017

EHNRI (2003) : Study on knowledge, attitude and dog ownership patterns related to rabies prevention in Addis Ababa, Ethiopia

Ethiopian Journal of Health Development, 16 (1), 105-112. [Google Scholar]

Ethiopian Public Health Institute (EPHI), Zoonosis Research Unit,Addis Ababa, Ethiopia

Faculty of Veterinary Medicine, Addis Ababa University, Debre-Zeit,Ethiopia

Mengistu F,Hussein K, Getahun G,Deressa, Yimer E,et al (2010) : Overview of Rabies in and Around Addis Ababa, in animals examined in EHNRI, Zoonosis Laboratory. Ethiopian Veterinary Journal, 14 (2), 91-101. [Google Scholar]

Moges N. (2015): Rabies in Ethiopia: ReviewArticle. Academic Journal of Animal Diseases 4(2):74-81, 2015. [Google Scholar

WHO (2017) Human Rabies: 2016 updates and call for data. Wkly Epidemiol Rec, 2017. 92(7):p.77-86 [PubMed]. [Google Scholar]

Yimer E,Newayeselassie B, Tefera G,Mekonnen Y,Bogale Y, et al (2002) Situation of Rabies in Ethiopia: A Retrospective study 1990-2000.

\section{Acknowledgements:}

Thanks go to the technical team of the animal health unit for discharging its tasks as well as the administrative staff members of Gulele Sub-city for their cooperation and administrative support.

The dedication of dog owners for provision of their dogs for immunization program from long distance and unbearablesituation is highly appreciated. 